\title{
A NEW APPROACH FOR CALCULATING THE ACCESSIBLE VOLUME IN EQUATIONS OF STATE FOR MIXTURES. II. APPLICATION TO LENNARD-JONES MIXTURES
}

\author{
B. SMIT and K.R. COX ${ }^{\star}$ \\ Koninklijke/Shell-Laboratorium, Amsterdam Shell Research B.V., P.O. Box 3003, \\ 1003 AA Amsterdam, (The Netherlands)
}

(November 10, 1987; accepted in final form March 18, 1988)

\begin{abstract}
Smit, B. and Cox, K.R., 1988. A new approach for calculating the accessible volume in equations of state. II. Application to Lennard-Jones mixtures. Fluid Phase Equilibria, 43: 181-189.

A new approach for calculating the accessible volume in the van der Waals equation of state has been used to calculate the Henry coefficient of Lennard-Jones mixtures. In these mixtures the size and energy parameters are varied. The results of these calculations are compared with calculations based on conformal solution theory and data from molecular dynamics simulations. This new approach gives a considerable improvement in the description of the data obtained from molecular dynamics simulation.
\end{abstract}

\section{INTRODUCTION}

One of the interesting problems in applied thermodynamics is the prediction of the properties of mixtures. Owing to the success of the theory of corresponding states in the prediction of the properties of simple pure fluids the extension of this theory to mixtures (conformal solution theory) has received much attention over the past few years. One of the important problems in the conformal solution theory is the development of appropriate mixing rules to relate the parameters of the reference fluid to the parameters of the mixtures (Prausnitz, 1969). Recent observations based on data of real fluids and computer simulations (Mentzer et al., 1981; Hoheisel et al., 1983) demonstrated that the conformal solution theory with the van der Waals mixing rules fails to describe the behaviour of mixtures with large size and

\footnotetext{
* Present address: Shell Development Company, Westhollow Research Center, P.O. Box 1380, Houston, TX $7751-1380$, U.S.A. 
energy differences. Several new mixing rules are proposed to take these differences into account (Hu et al., 1984; Ely, 1986; Jonah, 1986; Hamad and Mansoori 1987; Hamad et al., 1987).

Most of the currently-used mixing rules for the $b$ (volume) parameter in an equation of state are based on a hard-spheres model. In a recent publication Hamad et al. (1987) used the Kirkwood-Buff solution theory to develop a new set of density and temperature dependent mixing rules. In this work they proposed a new expression for the mixing rule for the $b$ parameter.

In a previous paper (Smit and Cox, 1988, this volume) we have suggested a new approach for calculating the accessible volume in equations of state for mixtures. In this paper we apply this approach to calculate the Henry coefficient for a binary mixture of Lennard--Jones fluids, in which the energy of interaction and the molecular sizes are varied. These calculations are compared with data from computer simulations (Shing and Gubbins; 1982) and with calculations based on the conformal solution theory with the new mixing rules of Hamad et al. (1987).

\section{THEORY}

\section{Equation of state}

In a previous paper (Smit and Cox, 1988) we have given a detailed description of the implementation of this new concept for calculating the accessible volume in an equation of state. Therefore, we will give in this section only a brief summary.

The normal mixing rule for the $b$ parameter in the van der Waals equation of state is based on a hard-spheres model. In such a model the accessible volume is estimated exclusively from pure component data. As soon as we consider a system in which specific binary interactions largely influence the volume occupied by the molecules a hard-spheres model leads to non-realistic results (Smit and Cox, 1988).

This inconsistency in the van der Waals equation can be resolved if we adopt the concept of apparent size as probed by a particular molecule. This concept states that the accessible volume of a particle $i$ is the total volume minus of the total apparent excluded volume of the particles $j$ as probed by particle $i$. Implementation of this concept in the van der Waals equation of state gives

$$
\begin{aligned}
& p / R T=\sum_{i} x_{i} p_{i} / R T \\
& p_{i} / R T=1 /\left(v-\sum_{j} x_{j} b_{i j}\right)-\sum_{j} x_{j} a_{i j} / R T v^{2}
\end{aligned}
$$


where $b_{i j}$ indicates the apparent volume of a $j$-particle as probed by an $i$-particle.

Henry coefficient

To derive the Henry coefficient we have used the following relation (Prausnitz, 1969)

$\ln \left(H_{i} / \rho k T\right)=\lim _{x_{i} \rightarrow 0} \ln \left(f_{i} / x_{i} \rho k T\right)$

where $H_{j}$ is the Henry coefficient, $f_{i}$ the fugacity and $x_{i}$ the mole fraction of component $i$ in the mixture. The fugacity can be calculated from

$R T \ln \left(f_{i} / x_{i} p\right)=\int_{V}^{\infty}\left[\left(\partial P / \partial N_{i}\right)_{T, V, N_{j}}-R T / V\right] \mathrm{d} V-R T \ln \left(V_{\mathrm{p}} / N R T\right)$

Substitution of eqn. (1) in eqns. (2) and (3) gives for the Henry coefficient of component 1 of a binary mixture

$\ln \left(H_{1}^{*} / \rho^{*} T^{*}\right)=-2 a_{12} / T^{*} v^{*}+b_{21} /\left(v^{*}-b_{22}\right)+\ln \left(v^{*} /\left(v^{*}-b_{12}\right)\right)$

In order to compare the calculations with molecular dynamics simulations, we have adopted reduced units $\left(T^{*}=k T / \epsilon\right.$ and $\left.\rho^{*}=\rho \sigma^{3}\right)$. Note that in this equation the van der Waals constants $a$ and $b$ have to be expressed in reduced units.

For comparison we also give the expression for the Henry coefficient of component 1 from the van der Waals equation with the hard-spheres mixing rule of the $b$ parameter:

$\ln \left(H_{1}^{*} / \rho^{*} T^{*}\right)=-2 a_{12} / T^{*} v^{*}+b_{11} /\left(v^{*}-b_{22}\right)+\ln \left(v^{*} /\left(v^{*}-b_{22}\right)\right)$

\section{CALCULATIONS AND RESULTS}

In order to apply the expressions for the Henry coefficients (eqns. (4) and (5)) to a mixture of Lennard-Jones fluids we have to estimate the $a$ and $b$ parameters. The $a_{12}$ and $b_{12}$ parameters are calculated from (Prausnitz, 1969)

$a_{12}=a_{22}\left(\epsilon_{12} / \epsilon_{22}\right)\left(d_{12} / d_{22}\right)^{3}$

$b_{12}=b_{22}\left(d_{12} / d_{22}\right)^{3}$

where $\epsilon_{i j}$ is the characteristic energy parameter of the intermolecular potential (Lennard-Jones) and $d_{i j}$ is the effective hard-sphere diameter of particle $j$ as probed by particle $i$. For a given potential Barker and Henderson (1967) have defined an effective hard-sphere diameter $d_{12}$ as

$d_{12}=\int_{0}^{\alpha}\left\{1-\exp \left[-\beta U_{12}^{*}(r)\right]\right\} \mathrm{d} r$

where $\sigma$ is the value of $r$ for which $U_{12}^{*}(r)$ is zero and $\beta^{*}$ is $1 / T^{*}$. 
Note that with these definitions $a_{12}$ and $b_{12}$ both approach zero if the intermolecular potential which describes the 1-2 interaction vanishes.

In the computer simulations (Shing and Gubbins, 1982) the Henry coefficient is calculated for a mixture of Lennard-Jones fluids with the same energy of interaction $\left(\epsilon_{12}\right)$ but different size ratios $\left(\sigma_{12}\right)$ and the same molecular sizes but different energy of interaction. These variations in energy and size are expressed in the parameters

$f_{\mathrm{r}} \equiv \epsilon_{12} / \epsilon_{22}$

$h_{\mathrm{r}} \equiv\left(\sigma_{12} / \sigma_{22}\right)^{3}$

Inspection of the expressions for $a_{12}$ and $b_{12}$ (eqns. (6) and (7)) shows that $f_{\mathrm{r}}$ can be substituted in the expression for $a_{12}$. Note that variation of $f_{\mathrm{r}}$ also affects the effective diameter due to the appearance of $\epsilon_{12}$ in the intermolecular (Lennard-Jones) potential. Variation of $h_{\mathrm{r}}$ directly affects the effective diameter. The definition of the effective hard-sphere diameter (eqn. (8)) shows that at constant $f_{\mathrm{r}}$ a different $\sigma_{12}$ can be regarded as a scaling of the coordinates, therefore

$d_{12} / d_{22}=\sigma_{12} / \sigma_{22}$

Thus we find for constant $f_{\mathrm{r}}$

$a_{12}=a_{22} h_{\mathrm{r}} f_{\mathrm{r}}$

$b_{12}=b_{22} h_{\mathrm{r}}$

The value for the $a_{22}$ and $b_{22}$ of the pure Lennard-Jones fluid can in principle be calculated from various properties of a pure Lennard-Jones fluid (e.g. from the critical data, fitted to the equation of state as proposed by Nicolas et al. (1979)). However, different properties will all give different sets of values for these parameters because the van der Waals equation is too "simple" to describe quantitatively the behaviour of a pure Lennard-Jones fluid. At this moment we are mainly interested in the influence of the new concept for calculating the accessible volume. Therefore, we have estimated $a_{22}$ and $b_{22}$ from the data obtained by computer simulations (Shing and Gubbins, 1982).

Note that for $h_{\mathrm{r}}=1$ and $f_{\mathrm{r}}=1$ the mixture reduces to a pure Lennard-Jones fluid and the new expression for the Henry coefficient (eqn. (4)) becomes identical to the expression based on the ordinary van der Waals equation (eqn. (5))

$\ln \left(H^{*} / \rho^{*} T^{*}\right)=-2 a / T^{*} v^{*}+b /\left(v^{*}-b\right)+\ln \left(v^{*} /\left(v^{*}-b\right)\right)$

If we consider the limit $f_{\mathrm{r}} \rightarrow \infty$ at constant $h_{\mathrm{r}}$, the values of the Henry coefficient as given by eqns. (4) or (5) approach a straight line (see Fig. 1) with a slope $(A)$ given by

$A=-2 a_{22}\left(d_{12} / d_{22}\right)^{3} / v^{*} T^{*}$ 


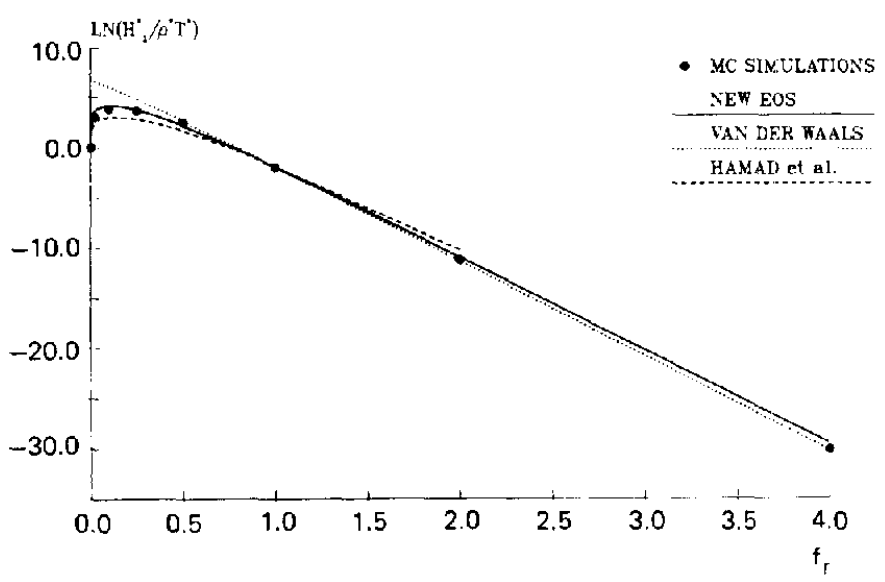

Fig. 1. Variation of the reduced Henry constant with $f_{\mathrm{r}}=\epsilon_{12} / \epsilon_{22}$.

In this limit the effective hard-sphere diameter $\left(d_{12} / \sigma\right)$ is 1 (cf. eqn. (8)), giving

$\left(d_{12} / d_{22}\right)^{3}=1.0116$

Thus we find for the slope

$A=-2 a_{22} 1.0116 / v^{*} T^{*}$

The data from the computer simulations (Shing and Gubbins, 1982) (Fig. 1) are used to estimate this slope and $a_{22}$ is calculated from eqn. 16 . This value for $a_{22}$ with the value of $H^{*}$ for the pure component (cf. Fig. 1) is used in

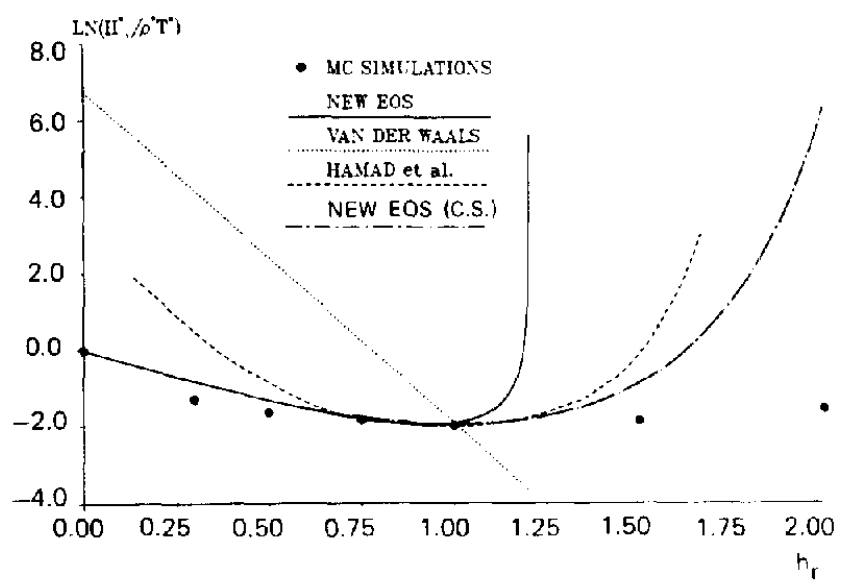

Fig. 2. Variation of the reduced Henry constant with $h_{\mathrm{r}}=\left(\sigma_{12} / \sigma_{22}\right)^{3}$. 
eqn. (14) to obtain a value for $b_{22}$

$a_{22}=7.44$ and $b_{22}=1.19$ (in dimensionless units)

With these values for $a_{22}$ and $b_{22}$ we have calculated the Henry coefficients with the ordinary van der Waals equation (eqn. (5)) and with the new equation (eqn. (4)) for different values of $h_{\mathrm{r}}$ and $f_{\mathrm{r}}$. The results are presented in Figs. 1 and 2. In these figures we have compared our calculations with data from computer simulations (Shing and Gubbins, 1982) and with calculations based on the mixing rules of Hamad et al. (1987).

\section{DISCUSSION AND CONCLUSIONS}

Figure 1 demonstrates clearly that the normal van der Waals equation with the hard-sphere mixing rules fails to describe the behaviour of this system at low values of $f_{\mathrm{r}}$. The new expression of the Henry coefficient based on the concept of apparent size gives a remarkable improvement in the description for low values of $f_{\mathrm{r}}$. Direct comparison with the calculations based on the mixing rules of Hamad et al. (1987) is not possible. Although we have used a much simpler formalism to describe the behaviour of this system we had to obtain the coefficients of the van der Waals equation from the behaviour of this system in the limit $f_{\mathrm{r}} \rightarrow \infty$. This will, of course, give a better description for high values of $f_{\mathrm{r}}$.

It is well known that the normal van der Waals equation with the hard-sphere mixing rules cannot describe the behaviour of mixtures with large differences in size of the components (Ely, 1986), as is demonstrated in Fig. 2. This figure points out dramatically the incorrect behaviour for $h_{\mathrm{r}}=0$. In this particular case a particle is added which does not interact with the solvent and therefore shows ideal behaviour. The new expression for the Henry coefficient gives a correct behaviour in this limit. Although the data from the simulations are not described exactly this new expression is giving a better description of these data for $h_{\mathrm{r}} \leq 1$ than the calculations based on the mixing rules of Hamad et al. (1987). The latter fail to give the correct behaviour for $h_{\mathrm{r}}=0$.

For values of $h_{\mathrm{r}}$ greater than unity the calculation based on both the mixing rules of Hamad et al. (1987) and the new expression predicts a Henry coefficient which tends to infinity. The reason for this becomes clear if we consider the new expression for the Henry coefficient (eqn. (4)) for $f_{\mathrm{r}}=1$ and variable $h_{\mathrm{r}}$

$$
\begin{aligned}
\ln \left(H_{1}^{*} / \rho^{*} T^{*}\right)= & -2 a_{22} h_{\mathrm{r}} / T^{*} v^{*}+b_{22} h_{\mathrm{r}} /\left(v^{*}-b_{22}\right) \\
& +\ln \left(v^{*} /\left(v^{*}-b_{22} h_{\mathrm{r}}\right)\right)
\end{aligned}
$$

The logarithm in the last term of eqn. (17) on the right-hand side demands $b_{12}=b_{22} h_{\mathrm{r}} \leq v^{*}$ 
This physical implication is that either infinite volume or infinite energy is required to insert a single particle which is too big according to the criterion above.

However, by using the van der Waals equation one excludes other than binary encounters in the estimates for the excluded volume. This overlap problem will become important as a large particle is inserted. Therefore, for high values of $h_{\mathrm{r}}$ the calculations become unreliable.

For a hard-spheres fluid the Carnahan-Starling equation (Carnahan and Starling, 1969; Dimitrilis and Prausnitz, 1986) takes into account this overlap problem. It is therefore interesting to replace the repulsive (hardspheres) term of the van der Waals equation by the Carnahan-Starling expression and implement the concept of apparent size. This gives for the pressure of a mixture:

$$
\begin{aligned}
& p / R T=\sum_{i} x_{i} p_{i} / R T \\
& p_{i} / R T=(1 / v)\left(1+y_{i}+y_{i}^{2}-y_{i}^{3}\right) /\left(1-y_{i}\right)^{3}-\sum_{j} x_{j} a_{i j} / R T v^{2} \\
& y_{i}=(1 / 4 v) \sum_{j} x_{j} b_{i j}
\end{aligned}
$$

With this expression we have obtained an expression for the Henry coefficient in which the values for $a$ and of the pure fluid are calculated in the same way as in eqn. (14) $(a=7.44$ and $b=0.503)$. The values of the Henry coefficient as a function of $f_{\mathrm{r}}$ obtained with this expression showed no significant deviations from the results obtained from the original expression (eqn. (4)). In the case that the particles differ in size, the introduction of the Carnahan-Starling expression gives a significant improvement for values of $h_{\mathrm{r}}$ greater than 1 .

This new approach for calculating the accessible volume appears to give a significant improvement in the description of data of computer simulations. Previous attempts (Hamad et al. 1987; Shing and Gubbins, 1982, 1983) to describe these data failed to give a correct limiting behaviour. Since we can explain the behaviour with a very simple concept and a simple equation of state it is our belief that this approach can contribute to a better understanding of the complex behaviour of mixtures.

\section{LIST OF SYMBOLS}

$a$ energy parameter of the van der Waals equation of state *

$b$ volume parameter of the van der Waals equation of state *

\footnotetext{
* In eqn (1) the van der Waals constants have the normal units; in all further equations these constants have reduced units.
} 
$d_{i j}$ effective hard-sphere diameter

$f_{\mathrm{r}}$ energy ratio

$f_{i} \quad$ fugacity of component $i$

$h_{\mathrm{r}}$ size ratio

$H_{i} \quad$ Henry coefficient of component $i$

$N$ number of particles

$p$ pressure

$p_{i} \quad$ partial pressure of component $i$

$r$ distance

$R$ gas constant

$T$ temperature

$U$ potential energy

$v$ molar volume

$V$ total volume

$y=b / 4 v$

$x_{i} \quad$ mole fraction of component $i$

\section{Greek letters}

$\beta$ reciprocal temperature

$\epsilon_{i j} \quad$ characteristic energy parameter for the $i-j$ interactions

$\rho$ density

$\sigma_{i j} \quad$ characteristic distance parameter for the $i-j$ interactions

\section{Superscript}

* denotes reduced units, which are conventional in molecular dynamics calculations, where $\epsilon$ is the unit of energy and $\sigma$ the unit of length.

\section{REFERENCES}

Barker, J.A. and D. Henderson, D., 1967. Perturbation theory and equations of state for fluids. II. A successful theory of liquids. J. Chem. Phys., 47: 4714-4721.

Ely, J.F., 1986. Improved mixing rules for one-fluid conformal solution calculations. In: K.C. Chao and R.L. Robinson, Jr. (Editors), Equations of State: Theory and Applications. ACS Symposium Series No. 300 American Chemiçal Society, Washington D.C., pp. 331-350.

Carnahan, N.F. and Starling, K.E., 1969. Equation of state for nonattracting rigid spheres. J. Chem. Phys., 51: 635-636.

Dimitrelis, D. and Prausnitz, J.M., 1986. Comparison of two hard-sphere reference systems for perturbation theories for mixtures. Fluid Phase Equilibria, 31: 1-21.

Hamad, E.Z. and Mansoori, G.A., 1987. Dense fluid theory of mixtures. J. Chem. Phys., 87: 6046-6052.

Hamad, E.Z., Mansoori, G.A. and Ely, J.F., 1987. Conformality in the Kirkwood-Buff solution theory of statistical mechanics. J. Chem. Phys., 86: 1478-1484. 
Hoheisel, C., Deiters, U. and Lucas, K., 1983. The extension of pure fluid thermodynamic properties to supercritical mixtures. Mol. Phys., 49: 159-170.

Hu. Y., Ludecke, D. and Prausnitz, J.M., 1984. Molecular thermodynamics of fluid mixtures containing molecules differing in size and potential energy. Fluid Phase Equilibria, 17: $217-241$.

Jonah, D.A., 1986. Consideration of some dilute-solution phenomena based on an expression for the Gibbs free energy. Int. J. Thermophysics, 7: 935-948.

Mentzer, R.A., Greenkorn, R.A. and Chao, K.A., 1981. Principle of corresponding states and vapor-liquid equilibria of molecular fluids, and their mixtures with light gases. Ind. Eng. Chem. Process Des. Dev., 20: 240-252.

Nicolas, J.J., Gubbins, K.E., Streett, W.B. and Tildesley, D.J., 1979. Equation of state for the Lennard-Jones fluid. Mol. Phys., 37: 1429-1454.

Prausnitz, J.M., 1969. Molecular Thermodynamics of Fluid-Phase Equilibria. Prentice-Hall, Englewood Cliffs.

Shing, K.S. and Gubbins, K.E., 1982. The chemical potential in dense fluids and fluid mixtures via computer simulation. Mol. Phys., 46: 1109-1128.

Shing, K.S. and Gubbins, K.E., 1983. The chemical potential in non-ideal liquid mixtures computer simulation and theory. Mol. Phys., 49: 1121-1138.

Smit, B. and Cox, K.R., 1988. A new approach for calculating the accessible volume in equations of state for mixtures. I. Theory and implementation in the van der Waals equation of state. Fluid Phase Equilibria, 43: 171-180. 